\title{
Gelfand-Shilov Smoothing Effect for the Radially Symmetric Spatially Homogeneous Landau Equation under the Hard Potential $\gamma=2$
}

\section{Haoguang* and WANG Hengyue}

School of Mathematics and Statistics, South-Central University for Nationalities, Wuhan 430074, China.

Received 12 December 2020; Accepted 3 July 2021

\begin{abstract}
Based on the spectral decomposition for the linear and nonlinear radially symmetric homogeneous non-cutoff Landau operators under the hard potential $\gamma=2$ in perturbation framework, we prove the existence and Gelfand-Shilov smoothing effect for solution to the Cauchy problem of the symmetric homogenous Landau equation with small initial datum.
\end{abstract}

AMS Subject Classifications: 35B65, 35E15, 76P05.

Chinese Library Classifications: O1.

Key Words: Gelfand-Shilov smoothing effect; spectral decomposition; Landau equation; hard potential $\gamma=2$.

\section{Introduction}

In this work, we consider the spatially homogeneous Landau equation

$$
\left\{\begin{array}{l}
\partial_{t} f=Q_{L}(f, f), \\
\left.f\right|_{t=0}=f_{0} \geq 0,
\end{array}\right.
$$

where $f=f(t, v)$ is the density distribution function depending on the variables $v \in \mathbb{R}^{3}$ and the time $t \geq 0$. The Landau bilinear collision operator is given by

$$
Q_{L}(g, f)(v)=\nabla_{v} \cdot \int_{\mathbb{R}^{3}} a\left(v-v_{*}\right)\left(g\left(v_{*}\right)\left(\nabla_{v} f\right)(v)-\left(\nabla_{v} g\right)\left(v_{*}\right) f(v)\right) \mathrm{d} v_{*},
$$

\footnotetext{
${ }^{*}$ Corresponding author. Email addresses: lihaoguang@scuec.edu.cn (H. G. Li), wanghengyue@mail.scuec . edu.cn (H. Y. Wang)
} 
where $a=\left(a_{i, j}\right)_{1 \leq i, j \leq 3}$ stands for the nonnegative symmetric matrix

$$
a(v)=\left(|v|^{2} \mathbf{I}-v \otimes v\right)|v|^{\gamma} \in M_{3}(\mathbb{R}), \quad-3<\gamma<+\infty .
$$

This equation is obtained as a limit of the Boltzmann equation, when all the collisions become grazing. See [1,2]. We shall consider the Cauchy problem of radially symmetric homogeneous non-cutoff Landau equation under the hard potential case $\gamma=2$ with the natural initial datum $f_{0} \geq 0$

$$
\int_{\mathbb{R}^{3}} f_{0}(v) \mathrm{d} v=1 ; \quad \int_{\mathbb{R}^{3}} v_{j} f_{0}(v) \mathrm{d} v=0, \quad j=1,2,3 ; \quad \int_{\mathbb{R}^{3}}|v|^{2} f_{0}(v) \mathrm{d} v=3 .
$$

Consider the fluctuation $f(t, v)=\mu(v)+\sqrt{\mu}(v) g(t, v)$ near the absolute Maxwellian

$$
\mu(v)=(2 \pi)^{-\frac{3}{2}} e^{-\frac{|v|^{2}}{2}}
$$

the Cauchy problem is reduced to

$$
\left\{\begin{array}{l}
\partial_{t} g+\mathcal{L}(g)=\Gamma(g, g), \quad t>0, v \in \mathbb{R}^{3}, \\
\left.g\right|_{t=0}=g^{0}
\end{array}\right.
$$

with $g^{0}(v)=\mu^{-\frac{1}{2}} f_{0}(v)-\sqrt{\mu}(v)$, where

$$
\Gamma(g, g)=\mu^{-\frac{1}{2}} Q_{\mathcal{L}}(\sqrt{\mu} g, \sqrt{\mu} g), \quad \mathcal{L}(g)=-\mu^{-\frac{1}{2}}\left(Q_{\mathcal{L}}(\sqrt{\mu} g, \mu)+Q_{\mathcal{L}}(\mu, \sqrt{\mu} g)\right) .
$$

The linear operator $\mathcal{L}$ is nonnegative with the null space

$$
\mathcal{N}=\operatorname{span}\left\{\sqrt{\mu}, \sqrt{\mu} v_{1}, \sqrt{\mu} v_{2}, \sqrt{\mu} v_{3}, \sqrt{\mu}|v|^{2}\right\} .
$$

The projection function $\mathbf{P}: \mathcal{S}^{\prime}\left(\mathbb{R}^{3}\right) \rightarrow \mathcal{N}$ is well defined. The assumption of the initial datum $f_{0}$ in (1.1), transforms to be $g^{0} \in \mathcal{N}^{\perp}$. We introduce the symmetric Gelfand-Shilov spaces, for $0<s \leq 1$,

$$
S_{\frac{1}{2 s}}^{\frac{1}{2 s}}\left(\mathbb{R}^{3}\right)=\left\{u \in \mathcal{S}^{\prime}\left(\mathbb{R}^{3}\right) ; \exists c>0, e^{c \mathcal{H}^{s}} u \in L^{2}\left(\mathbb{R}^{3}\right)\right\} ;
$$

where $\mathcal{H}=-\Delta+\frac{|v|^{2}}{4}$. This spaces can be also characterized through the decomposition into the Hermite basis $\left(\Psi_{\alpha}\right)_{\alpha \in \mathbb{N}^{3}}$,

$$
f \in S_{\frac{1}{2 s}}^{\frac{1}{2 s}}\left(\mathbb{R}^{3}\right) \Leftrightarrow f \in L^{2}\left(\mathbb{R}^{3}\right), \exists \epsilon_{0}>0,\left\|\left(e^{\epsilon_{0}|\alpha|^{s}}\left(f, \Psi_{\alpha}\right)_{L^{2}}\right)_{\alpha \in \mathbb{N}^{3}}\right\|_{l^{2}}<+\infty .
$$

For more details, see in [3, Theorem 2.1] or in [4, Proposition 2.1].

The existence and regularity of the solution to Cauchy problem for the spatially homogeneous Landau equation with hard potentials has already been treated in $[5,6]$. We 
can also refer to $[7,8]$ for the Gevrey regularity and Analytic smoothing effect for the solution of the Landau equation with hard potential. However, the mathematical technique in these works are mostly the energy methods. In this method, the smoothness effect of the Landau equation are almost not better than analytic smoothing effect.

In 2012, Lerner, Morimoto, Pravda-Starov, Xu(LMPX) began to study the radially symmetric spatially homogeneous non-cutoff Boltzmann equation with Maxwellian mole cules by using spectrum analysis and then showed that the solution enjoys the GelfandShilov smoothing effect in [9,10] and [11]. For Landau equation, Villani in [2] constructed a linear equation for the homogeneous Landau equation and MPX in [12] proved that the solution enjoys a Gelfand-Shilov regularizing effect in the class $S_{1 / 2}^{1 / 2}\left(\mathbb{R}^{3}\right)$. Recently, Li and $\mathrm{Xu}$ in [13] showed that global existence and Gelfand-Shilov regularizing properties of the solution to the Cauchy problem (1.2) for homogeneous non-cutoff Landau equation in Maxwellian molecules. From now on, the Gelfand-Shilov smoothing effect have never been studied in the non-Maxwellian case. In this paper, based on the spectral decomposition for the linear and nonlinear radially symmetric homogeneous non-cutoff Landau operators under the the hard potential $\gamma=2$ in perturbation framework, we show the existence and the Gelfand-Shilov smoothing effcet for the solution to the Cauchy problem for this spatially homogeneous Landau equation.

The main theorem is given in the following.

Theorem 1.1. There exists a small positive constant $\epsilon_{0}>0$, such that for any initial datum $g_{0} \in L^{2}\left(\mathbb{R}^{3}\right) \cap \mathcal{N}^{\perp}$ with

$$
\left\|g_{0}\right\|_{L^{2}\left(\mathbb{R}^{3}\right)} \leq \epsilon_{0}
$$

the Cauchy problem (1.2) for radially symmetric homogeneous non-cutoff Landau equation in hard potential $\gamma=2$ admits a global radially symmetric weak solution

$$
g \in L^{+\infty}\left(\left[0,+\infty\left[; L^{2}\left(\mathbb{R}^{3}\right)\right) .\right.\right.
$$

Moreover, we have the Gelfand-Shilov $S_{1}^{1}\left(\mathbb{R}^{3}\right)$ smoothing effect of Cauchy problem, for any given $T>0$, there exists a positive constant $\delta>0$ such that for all $0 \leq t \leq T$,

$$
\left\|e^{\delta t \sqrt{\mathcal{H}}} g(t)\right\|_{L^{2}}^{2}+\int_{0}^{t}\left\|\mathcal{H}^{\frac{1}{2}} e^{\delta t \sqrt{\mathcal{H}}} g(\tau)\right\|_{L^{2}}^{2} \mathrm{~d} \tau \leq\left\|g_{0}\right\|_{L^{2}}^{2} .
$$

Remark 1.1. (1) The decompositions of the linear and nonlinear Landau operators under the hard potential $\gamma=2$ are the technical part in this paper, in fact, the algebra structure of the linear Landau operator $\mathcal{L}$ is different from the Maxwellian molecules in [2] or [13].

(2) We think the regularizing property in the hard potential $\gamma=2$ is similar to that in Maxwellian molecules. This work is a first step to study the Gelfand-Shilov smoothing effect for Landau equation in non-Maxwellian case. It is interesting to study more general case rather than the case of $\gamma=2$. 
The rest of the paper is arranged as follows: in Section 2, we introduce the spectral analysis of the linear and nonlinear Landau operators, and the formal solution of the Cauchy problem (1.2) by transforming the linearized Landau equation into an infinite system of ordinary differential equations, then we construct the solution to the Cauchy problem for linear Landau equation. In Section 3, we prove the main Theorem 1.1. The spectrum analysis of the linear and nonlinear Landau operator is the technique part, which will be presented in Section 4 .

\section{Spectral analysis and linear Landau equation}

\subsection{Spectral analysis}

Diagonalization of the linear operators. We recall that $\left\{\varphi_{n}\right\}$ constitute an orthonormal basis of $L_{\text {rad }}^{2}\left(\mathbb{R}^{3}\right)$, the radially symmetric function space (see [11]) with

$$
\varphi_{n}(v)=\sqrt{\frac{n !}{4 \sqrt{2} \pi \Gamma\left(n+\frac{3}{2}\right)}} L_{n}^{\left(\frac{1}{2}\right)}\left(\frac{|v|^{2}}{2}\right) e^{-\frac{|v|^{2}}{4}},
$$

where $\Gamma(\cdot)$ is the standard Gamma function, for any $x>0$,

$$
\Gamma(x)=\int_{0}^{+\infty} t^{x-1} e^{-x} \mathrm{~d} x,
$$

and the Laguerre polynomial $L_{n}^{(\alpha)}$ of order $\alpha$, degree $n$ read,

$$
L_{n}^{(\alpha)}(x)=\sum_{r=0}^{n}(-1)^{n-r} \frac{\Gamma(\alpha+n+1)}{r !(n-r) ! \Gamma(\alpha+n-r+1)} x^{n-r} .
$$

In particular,

$$
\begin{aligned}
& \varphi_{0}(v)=(2 \pi)^{-\frac{3}{4}} e^{-\frac{|v|^{2}}{4}}=\sqrt{\mu}, \\
& \varphi_{1}(v)=\sqrt{\frac{2}{3}}\left(\frac{3}{2}-\frac{|v|^{2}}{2}\right) \sqrt{\mu}, \\
& \varphi_{2}(v)=\sqrt{\frac{8}{15}}\left(\frac{15}{8}-\frac{5|v|^{2}}{4}+\frac{|v|^{4}}{8}\right) \sqrt{\mu} .
\end{aligned}
$$

Furthermore, we have, for suitable radially symmetric function $g$,

$$
\mathcal{H}(g)=\sum_{n=0}^{\infty}\left(2 n+\frac{3}{2}\right) g_{n} \varphi_{n}, \quad g_{n}=\left\langle g, \varphi_{n}\right\rangle .
$$

It follows that

$$
g \in S_{\frac{1}{2 s}}^{\frac{1}{2 s}}\left(\mathbb{R}^{3}\right) \Leftrightarrow g \in L^{2}\left(\mathbb{R}^{3}\right), \exists \epsilon_{0}>0, \sum_{n=0}^{\infty} e^{\epsilon_{0}\left(2 n+\frac{3}{2}\right)^{s}}\left|g_{n}\right|^{2}<+\infty .
$$


Triangular effect of the linear and non-linear operators. We study now the algebra property of the nonlinear operators $\Gamma\left(\varphi_{n}, \varphi_{m}\right)$, and then the linear operator

$$
\mathcal{L} \varphi_{n}=\mathcal{L}_{1} \varphi_{n}+\mathcal{L}_{2} \varphi_{n}=-\Gamma\left(\varphi_{0}, \varphi_{n}\right)-\Gamma\left(\varphi_{n}, \varphi_{0}\right) .
$$

Similar to the proof of [11, Lemma 3.3], we can prove the following triangular effect for the nonlinear Landau operators on the basis $\left\{\varphi_{n}\right\}$ in hard potential $\gamma=2$.

Proposition 2.1. Let $\Gamma$ be the nonlinear Landau operator, the following algebraic identities hold true,

$$
\begin{aligned}
& \Gamma\left(\varphi_{0}, \varphi_{m}\right)=8 m \sqrt{(m+1)\left(m+\frac{3}{2}\right)} \varphi_{m+1}-8 m(2 m+3) \varphi_{m}+8(m-1) \sqrt{m\left(m+\frac{1}{2}\right)} \varphi_{m-1} ; \\
& \begin{aligned}
\Gamma\left(\varphi_{1}, \varphi_{m}\right) & =-\frac{8}{3} \sqrt{6(m+1)(m+2)\left(m+\frac{3}{2}\right)\left(m+\frac{5}{2}\right)} \varphi_{m+2} \\
& \quad+\frac{8(2 m+5)}{3} \sqrt{6(m+1)\left(m+\frac{3}{2}\right)} \varphi_{m+1}-\frac{8 \sqrt{6}}{3} m(m-1) \varphi_{m} ;
\end{aligned} \\
& \Gamma\left(\varphi_{2}, \varphi_{m}\right)=-\frac{8 \sqrt{30}}{3} \sqrt{(m+1)\left(m+\frac{3}{2}\right)} \varphi_{m+1} ; \\
& \Gamma\left(\varphi_{n}, \varphi_{m}\right)=0, \quad n>2,
\end{aligned}
$$

where for convenience, we always set $\varphi_{-1} \equiv 0$.

This proposition play a crucial role in the proof of the global existence of the solutions to the Landau equation under the hard potential $\gamma=2$. We will give the proof of this proposition in the Section 4.

Remark 2.1. Obviously, we can deduce from Proposition 2.1 that, for $n \in \mathbb{N}$

$$
\mathcal{L}_{1} \varphi_{n}=-\Gamma\left(\varphi_{0}, \varphi_{n}\right)=-8 n \sqrt{(n+1)\left(n+\frac{3}{2}\right)} \varphi_{n+1}+8 n(2 n+3) \varphi_{n}-8(n-1) \sqrt{n\left(n+\frac{1}{2}\right)} \varphi_{n-1}
$$

with $\varphi_{-1}=0$ and

$$
\mathcal{L}_{2} \varphi_{n}=-\Gamma\left(\varphi_{n}, \varphi_{0}\right)=-\left(40 \varphi_{1}-8 \sqrt{5} \varphi_{2}\right) \delta_{1, n}+8 \sqrt{5} \varphi_{1} \delta_{2, n} .
$$

Then

$$
\begin{aligned}
\mathcal{L} \varphi_{n}= & \mathcal{L}_{1} \varphi_{n}+\mathcal{L}_{2} \varphi_{n}=-\left[\boldsymbol{\Gamma}\left(\varphi_{0}, \varphi_{n}\right)+\boldsymbol{\Gamma}\left(\varphi_{n}, \varphi_{0}\right)\right] \\
=-8 n & \sqrt{(n+1)(n+3 / 2)} \varphi_{n+1}+8 n(2 n+3) \varphi_{n}-8(n-1) \sqrt{n(n+1 / 2)} \varphi_{n-1} \\
& -\left(40 \varphi_{1}-8 \sqrt{5} \varphi_{2}\right) \delta_{1, n}+8 \sqrt{5} \varphi_{1} \delta_{2, n}
\end{aligned}
$$


which satisfies that $\mathcal{L} \varphi_{0}=\mathcal{L} \varphi_{1} \equiv 0$. Let $\mathbf{P}$ be the orthogonal Projection on $\mathcal{N}$. Moreover, we have

$$
\begin{aligned}
& \mathcal{L} \varphi_{2}=-8 \sqrt{42} \varphi_{3}+112 \varphi_{2} ; \\
& \mathcal{L} \varphi_{n}=-8 n \sqrt{(n+1)(n+3 / 2)} \varphi_{n+1}+8 n(2 n+3) \varphi_{n}-8(n-1) \sqrt{n(n+1 / 2)} \varphi_{n-1}, \text { for } n>2 .
\end{aligned}
$$

Then for radial symmetric function $g$, one can verify that

$$
\mathcal{L}(g)=\sum_{n=2}^{+\infty} g_{n}(t) \mathcal{L}\left(\varphi_{n}\right) \in \mathcal{N}^{\perp}
$$

\subsection{The Cauchy problem of linear Landau equation}

Now we solve explicitly the Cauchy problem (1.2) associated to the non-cutoff radial symmetric spatially homogeneous Landau equation with hard potential $\gamma=2$ for the initial radial data $g^{0} \in L^{2}\left(\mathbb{R}^{3}\right) \cap \mathcal{N}^{\perp}$.

We search a radial solution to the Cauchy problem (1.2) in the form

$$
g(t)=\sum_{n=0}^{+\infty} g_{n}(t) \varphi_{n} \text { with } g_{n}(t)=\left\langle g(t), \varphi_{n}\right\rangle
$$

with initial data

$$
\left.g\right|_{t=0}=g^{0}=\sum_{n=0}^{+\infty}\left\langle g^{0}, \varphi_{n}\right\rangle \varphi_{n} .
$$

Remark that $g^{0} \in L^{2}\left(\mathbb{R}^{3}\right) \cap \mathcal{N}^{\perp}$ is equivalent to $g^{0}$ radial and

$$
\left\|g^{0}\right\|_{L^{2}\left(\mathbb{R}^{3}\right)}^{2}=\sum_{n=2}^{+\infty}\left|g_{n}^{0}\right|^{2}<+\infty, \quad g_{n}^{0}=\left(g^{0}, \varphi_{n}\right)_{L^{2}\left(\mathbb{R}^{3}\right)} .
$$

For suitable radially symmetric function $g$, we have

$$
\boldsymbol{\Gamma}(g, g)=g_{0}(t) \sum_{n=0}^{+\infty} g_{n}(t) \boldsymbol{\Gamma}\left(\varphi_{0}, \varphi_{n}\right)+g_{1}(t) \sum_{n=0}^{+\infty} g_{n}(t) \boldsymbol{\Gamma}\left(\varphi_{1}, \varphi_{n}\right)+g_{2}(t) \sum_{n=0}^{+\infty} g_{n}(t) \boldsymbol{\Gamma}\left(\varphi_{2}, \varphi_{n}\right) .
$$

It follows from Proposition 2.1 that,

$$
\begin{aligned}
\Gamma(g, g)=g_{0}(t) & \left(\sum_{n=1}^{+\infty} g_{n-1}(t) 8(n-1) \sqrt{n\left(n+\frac{3}{2}\right)}\right. \\
& \left.-\sum_{n=0}^{+\infty}\left[8 n(2 n+3) g_{n}(t)+8 n \sqrt{(n+1)\left(n+\frac{3}{2}\right)} g_{n+1}(t)\right]\right) \varphi_{n}
\end{aligned}
$$




$$
\begin{aligned}
& +g_{1}(t)\left(-\sum_{n=2}^{+\infty} \frac{8}{3} \sqrt{6(n-1) n\left(n-\frac{1}{2}\right)\left(n+\frac{1}{2}\right)} g_{n-2}(t)\right. \\
& \left.+\sum_{n=1}^{\infty} \frac{8(2 n+3)}{3} \sqrt{6 n\left(n+\frac{1}{2}\right)} g_{n-1}(t)-\sum_{n=0}^{+\infty} \frac{8 \sqrt{6}}{3} n(n-1) g_{n}(t)\right) \varphi_{n} \\
& -g_{2}(t) \sum_{n=1}^{+\infty} \frac{8 \sqrt{30}}{3} \sqrt{n\left(n+\frac{1}{2}\right)} g_{n-1}(t) \varphi_{n} .
\end{aligned}
$$

For radially symmetric function $g$, we can deduce from (2.1) in Remark 2.1 that

$$
\left(\mathcal{L}(g), \varphi_{0}\right)_{L^{2}\left(\mathbb{R}^{3}\right)}=0, \quad\left(\mathcal{L}(g), \varphi_{1}\right)_{L^{2}\left(\mathbb{R}^{3}\right)}=0 .
$$

Formally, we take inner product with $\varphi_{n}$ on both sides of the Cauchy problem (1.2), we find that the functions $\left\{g_{n}(t)\right\}$ satisfy the following infinite system of the differential equations

$$
\left\{\begin{array}{l}
\partial_{t} g_{0}(t)=0 \\
\partial_{t} g_{1}(t)=0 \\
\partial_{t} g_{n}(t)+\sum_{k=2}^{+\infty} g_{k}(t)\left(L \varphi_{k}, \varphi_{n}\right),=\left(\Gamma(g, g), \varphi_{n}\right), \quad \forall n \geq 2 ; \\
g_{n}(0)=\left\langle g^{0}, \varphi_{n}\right\rangle=g_{n}^{0}, \quad \forall n \in \mathbb{N} .
\end{array}\right.
$$

Since $g^{0} \in \mathcal{N}^{\perp}$, then it is obviously that

$$
g_{0}(t) \equiv\left\langle g^{0}, \varphi_{0}\right\rangle=0 ; \quad g_{1}(t) \equiv\left\langle g^{0}, \varphi_{1}\right\rangle=0
$$

The nonlinear Landau term turns out to be

$$
\Gamma(g, g)=-g_{2}(t) \sum_{n=2}^{+\infty} \frac{8 \sqrt{30}}{3} \sqrt{n\left(n+\frac{1}{2}\right)} g_{n-1}(t) \varphi_{n} .
$$

Furthermore, for $n \geq 2$,

$$
\begin{gathered}
\sum_{k=2}^{+\infty} g_{k}(t)\left(L \varphi_{k}, \varphi_{n}\right)=-8(n-1) \sqrt{n\left(n+\frac{1}{2}\right)} g_{n-1}(t)+8 n(2 n+3) g_{n}(t) \\
-8 n \sqrt{(n+1)\left(n+\frac{3}{2}\right)} g_{n+1}(t) .
\end{gathered}
$$

The infinite system of the differential equations (2.2) reduces to be

$$
\left\{\begin{array}{l}
g_{0}(t) \equiv 0 ; \quad g_{1}(t) \equiv 0 \\
\partial_{t} g_{2}(t)+112 g_{2}(t)=8 \sqrt{42} g_{3}(t) \\
\partial_{t} g_{n}(t)+8 n(2 n+3) g_{n}(t)=8(n-1) \sqrt{n\left(n+\frac{1}{2}\right) g_{n-1}}(t) \\
\quad+8 n \sqrt{(n+1)\left(n+\frac{3}{2}\right)} g_{n+1}(t)-\frac{8 \sqrt{30}}{3} \sqrt{n\left(n+\frac{1}{2}\right)} g_{2}(t) g_{n-1}(t), \quad \forall n>2 \\
g_{n}(0)=\left\langle g^{0}, \varphi_{n}\right\rangle .
\end{array}\right.
$$


Now we prove the existence of weak solution to Cauchy problem of the following linear Landau equation.

Proposition 2.2. For any $f, g^{0} \in L^{2}\left(\mathbb{R}^{3}\right) \cap \mathcal{N}^{\perp}$, there exists $c_{0}>0$, such that $f$ satifies

$$
\left\|\mathbb{P}_{2} f\right\|_{L^{2}\left(\mathbb{R}^{3}\right)}<c_{0}
$$

where $\mathbb{P}_{2} f=f_{2} \varphi_{2}$, then the Cauchy problem

$$
\left\{\begin{array}{l}
\partial_{t} g(t)+\mathcal{L}_{1} g(t)=\Gamma(f, g(t))-\mathcal{L}_{2} f, \\
\left.g(t, v)\right|_{t=0}=g^{0}(v)
\end{array}\right.
$$

admits a weak solution

$$
g \in L^{+\infty}\left(\left[0,+\infty\left[, L^{2}\left(\mathbb{R}^{3}\right) \cap \mathcal{N}^{\perp}\right) \cap C^{0}\left(\left[0,+\infty\left[; \mathcal{S}^{\prime}\left(\mathbb{R}^{3}\right)\right) .\right.\right.\right.\right.
$$

Moreover, we have

$$
\|g\|_{L^{2}\left(\mathbb{R}^{3}\right)} \leq\left\|g^{0}\right\|_{L^{2}\left(\mathbb{R}^{3}\right)} .
$$

Before the proof of Proposition 2.2, we provide the sharp trilinear estimates for the radially symmetric nonlinear landau operator in the following Lemma.

Lemma 2.1. For all $f, g, h \in \mathcal{S}_{r}\left(\mathbb{R}^{3}\right) \cap \mathcal{N}^{\perp}$, we have

$$
\left|(\Gamma(f, g), h)_{L^{2}}\right| \leq 8\left\|\mathbb{P}_{2} f\right\|_{L^{2}}\left\|\mathcal{H}^{\frac{1}{2}} g\right\|_{L^{2}}\left\|\mathcal{H}^{\frac{1}{2}} h\right\|_{L^{2}} .
$$

Proof. Let $f, g, h \in \mathcal{S}_{r}\left(\mathbb{R}^{3}\right) \cap \mathcal{N}^{\perp}$ be some radially symmetric Schwartz functions, we can decompose these functions into the Hermite basis $\left(\varphi_{n}\right)_{n \geq 0}$ as follows

$$
f=\sum_{n=2}^{+\infty} f_{n} \varphi_{n}, \quad g=\sum_{n=2}^{+\infty} g_{n} \varphi_{n}, \quad h=\sum_{n=2}^{+\infty} h_{n} \varphi_{n} .
$$

It follows from Proposition 2.1 that,

$$
\boldsymbol{\Gamma}(f, g)=f_{2} \sum_{n=0}^{+\infty} g_{n} \Gamma\left(\varphi_{2}, \varphi_{n}\right)
$$

This implies that,

$$
(\Gamma(f, g), h)_{L^{2}}=-f_{2} \sum_{n=3}^{+\infty} \frac{8 \sqrt{30}}{3} \sqrt{n\left(n+\frac{1}{2}\right)} g_{n-1} h_{n} .
$$

By using the Cauchy-Schwartz inequality, one can verify that

$$
\left|(\Gamma(f, g), h)_{L^{2}}\right| \leq 8\left\|\mathbb{P}_{2} f\right\|_{L^{2}}\left\|\mathcal{H}^{\frac{1}{2}} g\right\|_{L^{2}}\left\|\mathcal{H}^{\frac{1}{2}} h\right\|_{L^{2}}
$$

This ends the proof of Lemma 2.1. 
Now we are prepared to prove the Proposition 2.2.

The proof of the Proposition 2.2. We search a radially symmetric solution to the Cauchy problem (2.5) in the form

$$
g(t)=\sum_{n=2}^{+\infty} g_{n}(t) \varphi_{n}
$$

with initial data $g(0)=g^{0}=\sum_{n=0}^{+\infty}\left(g^{0}, \varphi_{n}\right)_{L^{2}\left(\mathbb{R}^{3}\right)} \varphi_{n}$. Remark that $g^{0} \in L^{2}\left(\mathbb{R}^{3}\right) \cap \mathcal{N}^{\perp}$ is equivalent to $g^{0}$ radial and

$$
\left\|g^{0}\right\|_{L^{2}}^{2}=\sum_{n=2}^{+\infty}\left|g_{n}^{0}\right|^{2}<+\infty
$$

Since $f \in L^{2}\left(\mathbb{R}^{3}\right) \cap \mathcal{N}^{\perp}$, for radially symmetric function $g \in \mathcal{S}_{r}\left(\mathbb{R}^{3}\right) \cap \mathcal{N}^{\perp}$, it follows from Proposition 2.1 that,

$$
\Gamma(f, g)=-f_{2} \sum_{n=1}^{+\infty} \frac{8 \sqrt{30}}{3} \sqrt{n\left(n+\frac{1}{2}\right)} g_{n-1}(t) \varphi_{n} .
$$

Moreover, for this $g \in \mathcal{S}_{r}\left(\mathbb{R}^{3}\right) \cap \mathcal{N}^{\perp}$, we can deduce from Remark 2.1 that

$$
\begin{gathered}
\mathcal{L}_{1} g=\sum_{n=2}^{+\infty}\left(-8(n-1) \sqrt{n(n+1 / 2)} g_{n-1}(t)+8 n(2 n+3) g_{n}(t)\right. \\
\left.-8 n \sqrt{(n+1)(n+3 / 2)} g_{n+1}(t)\right) \varphi_{n},
\end{gathered}
$$

with $\varphi_{-1}=0$ and

$$
\mathcal{L}_{2} f=8 \sqrt{5} f_{2}(t) \varphi_{1}
$$

Formally, we take inner product with $\left\{\varphi_{n}\right\}_{n \geq 2}$ on both sides of the Cauchy problem (2.5), we find that the functions $\left\{g_{n}(t)\right\}_{n \geq 2}$ satisfy the following infinite system of the differential equations

$$
\left\{\begin{array}{l}
\partial_{t} g_{2}(t)+112 g_{2}(t)=8 \sqrt{42} g_{3}(t) \\
\partial_{t} g_{n}(t)+8 n(2 n+3) g_{n}(t)=8(n-1) \sqrt{n\left(n+\frac{1}{2}\right)} g_{n-1}(t) \\
\quad+8 n \sqrt{(n+1)\left(n+\frac{3}{2}\right)} g_{n+1}(t)-\frac{8 \sqrt{30}}{3} \sqrt{n\left(n+\frac{1}{2}\right)} f_{2} g_{n-1}(t), \quad \forall n \geq 3 \\
g_{n}(0)=\left\langle g^{0}, \varphi_{n}\right\rangle .
\end{array}\right.
$$

Let us now fix some positive integer $N \geq 2$ and define the following function $u_{N}:[0,+\infty[$ $\times \mathbb{R}^{3} \rightarrow \mathcal{S}^{\prime}\left(\mathbb{R}^{3}\right)$ by

$$
u_{N}(t)=\sum_{n=2}^{N} g_{n}(t) \varphi_{n}
$$


where $g_{n}(t)$ is the solution to the ODEs (2.6) for $n \leq N$. For $h \in \mathcal{S}^{\prime}\left(\mathbb{R}^{3}\right)$, we defined the $k+1$ projection $\mathbb{P}_{k}$ that

$$
\mathbb{P}_{k} h=\sum_{n=0}^{k}\left\langle h, \varphi_{n}\right\rangle \varphi_{n}
$$

Then for $N \geq 2, \mathbb{P}_{N} u_{N}=u_{N}$ and $u_{N}$ satisfies

$$
\left\{\begin{array}{l}
\partial_{t} u_{N}(t)+\mathbb{P}_{N} \mathcal{L}_{1} u_{N}(t)=\mathbb{P}_{N} \boldsymbol{\Gamma}\left(f, u_{N}\right), \\
u_{N}(0)=\sum_{n=2}^{N}\left\langle g^{0}, \varphi_{n}\right\rangle \varphi_{n} .
\end{array}\right.
$$

For $N \geq 2$, taking the inner product of $u_{N}(t)$ in $L^{2}\left(\mathbb{R}^{3}\right)$ on both sides of $(2.7)$, we have

$$
\left(\partial_{t} u_{N}(t), u_{N}(t)\right)_{L^{2}\left(\mathbb{R}^{3}\right)}+\left(\mathbb{P}_{N} \mathcal{L}_{1} u_{N}(t), u_{N}(t)\right)_{L^{2}\left(\mathbb{R}^{3}\right)}=\left(\mathbb{P}_{N} \Gamma\left(f, u_{N}(t)\right), u_{N}(t)\right)_{L^{2}\left(\mathbb{R}^{3}\right)} .
$$

Then one can verify from (2.6) that

$$
\begin{aligned}
& \frac{1}{2} \frac{\mathrm{d}}{\mathrm{d} t}\left\|u_{N}(t)\right\|_{L^{2}\left(\mathbb{R}^{3}\right)}^{2}+\sum_{k=2}^{N} 8 k(2 k+3)\left|g_{k}(t)\right|^{2} \\
= & 2 \sum_{k=2}^{N-1} 8 k \sqrt{(k+1)\left(k+\frac{3}{2}\right)} g_{k}(t) g_{k+1}(t)+\left(\mathbb{P}_{N} \Gamma\left(f, u_{N}(t)\right), u_{N}(t)\right)_{L^{2}\left(\mathbb{R}^{3}\right)} .
\end{aligned}
$$

By using Cauchy-Schwartz inequality, we have

$$
\begin{aligned}
& \sum_{k=2}^{N-1} 8 k \sqrt{(k+1)\left(k+\frac{3}{2}\right)} g_{k}(t) g_{k+1}(t) \\
\leq & \sum_{k=2}^{N-1} 4 k(k+3 / 2)\left|g_{k}(t)\right|^{2}+\sum_{k=2}^{N-1} 4 k(k+1)\left|g_{k+1}(t)\right|^{2} \\
\leq & \sum_{k=2}^{N-1} 4 k(2 k+1 / 2)\left|g_{k}(t)\right|^{2} .
\end{aligned}
$$

We can conclude from Lemma 2.1 that

$$
\frac{1}{2} \frac{\mathrm{d}}{\mathrm{d} t}\left\|u_{N}(t)\right\|_{L^{2}\left(\mathbb{R}^{3}\right)}^{2}+\left\|\mathcal{H}^{1 / 2} u_{N}(t)\right\|_{L^{2}\left(\mathbb{R}^{3}\right)}^{2} \leq 8\left\|\mathbb{P}_{2} f\right\|_{L^{2}\left(\mathbb{R}^{3}\right)}\left\|\mathcal{H}^{\frac{1}{2}} u_{N}(t)\right\|_{L^{2}\left(\mathbb{R}^{3}\right)}^{2} .
$$

By the assumption that $\left\|\mathbb{P}_{2} f\right\|_{L^{2}\left(\mathbb{R}^{3}\right)}<c_{0}$ with $c_{0}$ small, then there exists a positive constant $\delta_{0}>0$, such that for any $t>0$, we have

$$
\left\|u_{N}(t)\right\|_{L^{2}\left(\mathbb{R}^{3}\right)}^{2}+\delta_{0} \int_{0}^{t}\left\|\mathcal{H}^{1 / 2} u_{N}(\tau)\right\|_{L^{2}\left(\mathbb{R}^{3}\right)}^{2} \mathrm{~d} \tau \leq\left\|u_{N}(0)\right\|_{L^{2}\left(\mathbb{R}^{3}\right)}^{2} \leq\left\|g^{0}\right\|_{L^{2}\left(\mathbb{R}^{3}\right)}^{2} .
$$


For any $N \geq 2$, there exists positive constant $C>0$, we have also

$$
\begin{gathered}
\left\|\mathcal{H}^{-2} \mathbb{P}_{N} \mathcal{L}_{1}\left(u_{N}\right)\right\|_{L^{2}\left(\mathbb{R}^{3}\right)} \leq C\left\|g^{0}\right\|_{L^{2}\left(\mathbb{R}^{3}\right)}, \\
\left\|\mathcal{H}^{-1} \mathbb{P}_{N} \boldsymbol{\Gamma}\left(f, u_{N}\right)\right\|_{\left.L^{2}\left(\mathbb{R}^{3}\right)\right)} \leq C\left\|g^{0}\right\|_{L^{2}\left(\mathbb{R}^{3}\right)}^{2}
\end{gathered}
$$

So that Eq. (2.7) implies that the sequence $\left\{\frac{\mathrm{d}}{\mathrm{d} t} u_{N}(t)\right\}$ is uniformly bounded in $\mathcal{S}^{\prime}\left(\mathbb{R}^{3}\right)$ with respect to $N \in \mathbb{N}$ and $t \in[0,+\infty$. The Arzelà-Ascoli Theorem implies that, there exists a subsequence $\left\{u_{N_{k}}(t)\right\} \subset\left\{u_{N}(t)\right\}$ such that

$$
u_{N_{k}}(t) \rightarrow g(t) \in C^{0}\left(\left[0,+\infty\left[; \mathcal{S}^{\prime}\left(\mathbb{R}^{3}\right)\right) .\right.\right.
$$

Moreover, we have

$$
\|g(t)\|_{L^{2}\left(\mathbb{R}^{3}\right)} \leq \liminf _{k \rightarrow+\infty}\left\|u_{N_{k}}(t)\right\|_{L^{2}\left(\mathbb{R}^{3}\right)} \leq\left\|g^{0}\right\|_{L^{2}\left(\mathbb{R}^{3}\right)}
$$

This shows that

$$
g \in L^{+\infty}\left(\left[0,+\infty\left[, L^{2}\left(\mathbb{R}^{3}\right) \cap \mathcal{N}^{\perp}\right) \cap C^{0}\left(\left[0,+\infty\left[; \mathcal{S}^{\prime}\left(\mathbb{R}^{3}\right)\right),\right.\right.\right.\right.
$$

$g(t)$ is a weak solution of Cauchy problem (2.5). We end the proof of Proposition 2.2.

\section{The Proof of Theorem 1.1}

We recall the definition of weak solution of (1.2):

Definition 3.1. Let $g^{0} \in \mathcal{S}^{\prime}\left(\mathbb{R}^{3}\right), g(t, v)$ is called a weak solution of the Cauchy problem (1.2) if it satisfies the following conditions:

$$
\begin{aligned}
& g \in C^{0}\left(\left[0,+\infty\left[; \mathcal{S}^{\prime}\left(\mathbb{R}^{3}\right)\right), \quad g(0, v)=g^{0}(v),\right.\right. \\
& \mathcal{L}(g) \in L^{2}\left(\left[0, T\left[; \mathcal{S}^{\prime}\left(\mathbb{R}^{3}\right)\right), \quad \Gamma(g, g) \in L^{2}\left(\left[0, T\left[; \mathcal{S}^{\prime}\left(\mathbb{R}^{3}\right)\right), \quad \forall T>0,\right.\right.\right.\right. \\
& \langle g(t), \phi(t)\rangle-\left\langle g^{0}, \phi(0)\right\rangle+\int_{0}^{t}\langle\mathcal{L} g(\tau), \phi(\tau)\rangle \mathrm{d} \tau \\
= & \int_{0}^{t}\left\langle g(\tau), \partial_{\tau} \phi(\tau)\right\rangle \mathrm{d} \tau+\int_{0}^{t}\langle\boldsymbol{\Gamma}(g(\tau), g(\tau)), \phi(\tau)\rangle \mathrm{d} \tau, \quad \forall t \geq 0,
\end{aligned}
$$

for any $\phi(t) \in C^{1}\left(\left[0,+\infty\left[; \mathcal{S}\left(\mathbb{R}^{3}\right)\right)\right.\right.$.

Now we are prepared to prove Theorem 1.1.

Existence. By using Proposition 2.2, we begin to prove the Global existence of solutions to the nonlinear Landau equation. For linear Landau equation (2.5), we consider the following sequence of iterating approximate solutions:

$$
\left\{\begin{array}{l}
\partial_{t} g^{n+1}+\mathcal{L}_{1} g^{n+1}=\Gamma\left(g^{n}, g^{n+1}\right)-\mathcal{L}_{2} g^{n}, \quad t>0, v \in \mathbb{R}^{3}, \\
\left.g^{n+1}(t, v)\right|_{t=0}=g_{0}(v),
\end{array}\right.
$$


starting from $g^{0}(t, v) \equiv g_{0}(v)$. Taking $g=g^{n+1}, f=g^{n}$ in Proposition 2.2 gives, for any $t>0$, there exists a constant $\delta_{0}>0$,

$$
\left\|g^{n}\right\|_{L^{2}\left(\mathbb{R}^{3}\right)}^{2}+\delta_{0} \int_{0}^{t}\left\|\mathcal{H}^{1 / 2} g^{n}\right\|_{L^{2}\left(\mathbb{R}^{3}\right)}^{2} \mathrm{~d} \tau \leq\left\|g^{0}\right\|_{L^{2}\left(\mathbb{R}^{3}\right)}^{2} \leq \epsilon^{2}
$$

Then it remains to prove the convergence of the sequence

$$
\left\{g^{n}, n \in \mathbb{N}\right\} \subset L_{t}^{\infty}\left(L^{2}\left(\mathbb{R}^{3}\right) \cap \mathcal{N}^{\perp}\right), \quad\left\{\mathcal{H}^{1 / 2} g^{n}, n \in \mathbb{N}\right\} \subset L_{t}^{2}\left(L^{2}\left(\mathbb{R}^{3}\right)\right) .
$$

Set $w^{n}=g^{n+1}-g^{n}$, one can verify from (3.1) that

$$
\partial_{t} w^{n}+\mathcal{L}_{1} w^{n}=\boldsymbol{\Gamma}\left(g^{n}, w^{n}\right)+\boldsymbol{\Gamma}\left(w^{n-1}, g^{n}\right)-\mathcal{L}_{2} w^{n-1},
$$

with $\left.w^{n}\right|_{t=0}=0$. Since $w^{n} \in \mathcal{N}^{\perp}$, we have

$$
\mathcal{L}_{2} w^{n-1} \equiv 0 .
$$

By the similar computation as (2.8), we get

$$
\begin{aligned}
& \frac{1}{2} \frac{\mathrm{d}}{\mathrm{d} t}\left\|w^{n}(t)\right\|_{L^{2}\left(\mathbb{R}^{3}\right)}^{2}+\left\|\mathcal{H}^{1 / 2} w^{n}(t)\right\|_{L^{2}\left(\mathbb{R}^{3}\right)}^{2} \\
\leq & \left(\boldsymbol{\Gamma}\left(g^{n}, w^{n}\right), w^{n}\right)_{L^{2}\left(\mathbb{R}^{3}\right)}+\left(\boldsymbol{\Gamma}\left(w^{n-1}, g^{n}\right), w^{n}\right)_{L^{2}\left(\mathbb{R}^{3}\right)} .
\end{aligned}
$$

By using Lemma 2.1 again, we have

$$
\begin{aligned}
& \left|\left(\boldsymbol{\Gamma}\left(g^{n}, w^{n}\right), w^{n}\right)_{L^{2}\left(\mathbb{R}^{3}\right)}\right| \leq 8\left\|g^{0}\right\|_{L^{2}\left(\mathbb{R}^{3}\right)}\left\|\mathcal{H}^{\frac{1}{2}} w^{n}\right\|_{L^{2}\left(\mathbb{R}^{3}\right)}^{2} ; \\
& \left|\left(\boldsymbol{\Gamma}\left(w^{n-1}, g^{n}\right), w^{n}\right)_{L^{2}\left(\mathbb{R}^{3}\right)}\right| \leq 8\left\|w^{n-1}\right\|_{L^{2}\left(\mathbb{R}^{3}\right)}\left\|\mathcal{H}^{\frac{1}{2}} g^{n}\right\|_{L^{2}\left(\mathbb{R}^{3}\right)}\left\|\mathcal{H}^{\frac{1}{2}} w^{n}\right\|_{L^{2}\left(\mathbb{R}^{3}\right)} .
\end{aligned}
$$

For any $t \geq 0$, we obtain that,

$$
\begin{aligned}
&\left\|w^{n}(t)\right\|_{L^{2}\left(\mathbb{R}^{3}\right)}^{2}+\int_{0}^{t}\left\|\mathcal{H} w^{n}\right\|_{L^{2}\left(\mathbb{R}^{3}\right)}^{2} \mathrm{~d} \tau \\
& \leq 8\left\|w^{n-1}\right\|_{L_{t}^{\infty} L^{2}\left(\mathbb{R}^{3}\right)} \int_{0}^{t}\left\|\mathcal{H}^{\frac{1}{2}} g^{n}\right\|_{L^{2}\left(\mathbb{R}^{3}\right)}\left\|\mathcal{H}^{\frac{1}{2}} w^{n}\right\|_{L^{2}\left(\mathbb{R}^{3}\right)} \mathrm{d} \tau \\
& \leq \lambda\left\|w^{n-1}\right\|_{L_{t}^{\infty} L^{2}\left(\mathbb{R}^{3}\right)},
\end{aligned}
$$

for some $0<\lambda<1$. Then it follows that

$$
\left\|w^{n}(t)\right\|_{L_{t}^{\infty} L^{2}\left(\mathbb{R}^{3}\right)}^{2}+\int_{0}^{t}\left\|\mathcal{H} w^{n}\right\|_{L^{2}\left(\mathbb{R}^{3}\right)}^{2} \mathrm{~d} \tau \leq \lambda^{n-1}\left\|w^{1}\right\|_{L_{t}^{\infty} L^{2}\left(\mathbb{R}^{3}\right)} \leq 2 \lambda^{n-1}\left\|g^{0}\right\|_{L^{2}\left(\mathbb{R}^{3}\right)},
$$

for some $0<\lambda<1$. It concludes that $\left\{g^{n}\right\}$ is a Cauchy sequence which satisfies

$$
\left\{g^{n}, n \in \mathbb{N}\right\} \subset L_{t}^{\infty}\left(L^{2}\left(\mathbb{R}^{3}\right) \cap \mathcal{N}^{\perp}\right), \quad\left\{\mathcal{H} g^{n}, n \in \mathbb{N}\right\} \subset L_{t}^{2} L^{2}\left(\mathbb{R}^{3}\right) .
$$


And its limit function $g$ is a desired solution to the Cauchy problem (1.2) in $t \in[0,+\infty[$. We obtain the global solution

$$
g \in L^{+\infty}\left(\left[0,+\infty\left[, L^{2}\left(\mathbb{R}^{3}\right) \cap \mathcal{N}^{\perp}\right) \cap C^{0}\left(\left[0,+\infty\left[; \mathcal{S}^{\prime}\left(\mathbb{R}^{3}\right)\right) .\right.\right.\right.\right.
$$

Gelfand-Shilov smoothing effect. Let $0 \leq \delta, \delta_{1} \leq 1$. Define

$$
h=M_{\delta_{1}}(\delta t) g \text { with } M_{\delta_{1}}(\delta t)=\frac{e^{\delta t \sqrt{\mathcal{H}}}}{1+\delta_{1} e^{\delta t \sqrt{\mathcal{H}}}} .
$$

The function $h$ depends on $\delta, \delta_{1}$. We can also write that

$$
g=\left(M_{\delta_{1}}(\delta t)\right)^{-1} h=\delta_{1} h+e^{-\delta t \sqrt{\mathcal{H}}} h .
$$

The equation (1.2)

$$
\partial_{t} g+\mathcal{L} g=\Gamma(g, g)
$$

reads as

$$
\begin{aligned}
& \left(M_{\delta_{1}}(\delta t)\right)^{-1} \partial_{t} h+\mathcal{L}\left(\left(M_{\delta_{1}}(\delta t)\right)^{-1} h\right) \\
= & \Gamma\left(\left(M_{\delta_{1}}(\delta t)\right)^{-1} h,\left(M_{\delta_{1}}(\delta t)\right)^{-1} h\right)+\delta \sqrt{\mathcal{H}} e^{-\delta t \sqrt{\mathcal{H}}} h .
\end{aligned}
$$

It follows that

$$
\begin{aligned}
& \partial_{t} h+\left(M_{\delta_{1}}(\delta t)\right) \mathcal{L}\left(\left(M_{\delta_{1}}(\delta t)\right)^{-1} h\right) \\
= & \left(M_{\delta_{1}}(\delta t)\right) \Gamma\left(\left(M_{\delta_{1}}(\delta t)\right)^{-1} h,\left(M_{\delta_{1}}(\delta t)\right)^{-1} h\right)+\frac{\delta \sqrt{\mathcal{H}}}{1+\delta_{1} e^{\delta t \sqrt{\mathcal{H}}}} h .
\end{aligned}
$$

We define

$$
h_{\delta_{2}}=\left(1+\delta_{2} \mathcal{H}\right)^{-1} h \quad \text { with } \quad 0<\delta_{2} \leq 1 .
$$

Then by multiplying $\left(1+\delta_{2} \mathcal{H}\right)^{-2} h$ on both sides of (3.3), we have

$$
\begin{aligned}
& \frac{1}{2}\left\|h_{\delta_{2}}\right\|_{L^{2}}^{2}+\left(\left(1+\delta_{2} \mathcal{H}\right)^{-1}\left(M_{\delta_{1}}(\delta t)\right) \mathcal{L}\left(\left(M_{\delta_{1}}(\delta t)\right)^{-1} h\right), h_{\delta_{2}}\right) \\
&=\left(\left(1+\delta_{2} \mathcal{H}\right)^{-1}\left(M_{\delta_{1}}(\delta t)\right) \Gamma\left(\left(M_{\delta_{1}}(\delta t)\right)^{-1} h,\left(M_{\delta_{1}}(\delta t)\right)^{-1} h\right), h_{\delta_{2}}\right) \\
& \quad+\left\|\left(\frac{\delta \sqrt{\mathcal{H}}}{1+\delta_{1} e^{\delta t \sqrt{\mathcal{H}}}}\right)^{\frac{1}{2}} h_{\delta_{2}}\right\|_{L^{2}}^{2} .
\end{aligned}
$$

Set $h=\sum_{n=2}^{\infty} h_{n} \varphi_{n}$, then

$$
h_{\delta_{2}}=\sum_{n=2}^{\infty} h_{n, \delta_{2}} \varphi_{n}=\sum_{n=2}^{\infty} h_{n}\left(1+\delta_{2}\left(2 n+\frac{3}{2}\right)\right)^{-1} \varphi_{n}
$$


one can verify that

$$
\begin{aligned}
& \quad\left(M_{\delta_{1}}(\delta t)\right) \mathcal{L}\left(\left(M_{\delta_{1}}(\delta t)\right)^{-1} h\right) \\
& =\sum_{n=2}^{\infty} h_{n}(t) \frac{1+\delta_{1} e^{t \delta \sqrt{2 n+\frac{7}{2}}}}{e^{t \delta \sqrt{2 n+\frac{3}{2}}}}\left[-8 n \sqrt{(n+1)\left(n+\frac{3}{2}\right)} \frac{e^{t \delta \sqrt{2 n+\frac{7}{2}}}}{1+\delta_{1} e^{t \delta \sqrt{2 n+\frac{7}{2}}}} \varphi_{n+1}\right. \\
& \left.\quad+8 n(2 n+3) \frac{e^{t \delta \sqrt{2 n+\frac{3}{2}}}}{1+\delta_{1} e^{t \delta \sqrt{2 n+\frac{3}{2}}}} \varphi_{n}-8(n-1) \sqrt{n\left(n+\frac{1}{2}\right)} \frac{e^{t \delta \sqrt{2 n-\frac{1}{2}}}}{1+\delta_{1} e^{t \delta \sqrt{2 n-\frac{1}{2}}}} \varphi_{n-1}\right] .
\end{aligned}
$$

It follows from the Cauchy-Schwartz inequality that

$$
\begin{aligned}
& \left(\left(1+\delta_{2} \mathcal{H}\right)^{-1}\left(M_{\delta_{1}}(\delta t)\right) \mathcal{L}\left(\left(M_{\delta_{1}}(\delta t)\right)^{-1} h\right), h_{\delta_{2}}\right) \\
& =\sum_{n=2}^{\infty} 8 n(2 n+3)\left|h_{n, \delta_{2}}(t)\right|^{2}-\sum_{n=2}^{\infty} 8 n \sqrt{(n+1)\left(n+\frac{3}{2}\right)} h_{n, \delta_{2}}(t) h_{n+1, \delta_{2}}(t) \\
& \times\left[\frac{\left(1+\delta_{2}\left(2 n+\frac{3}{2}\right)\right) e^{\delta t \sqrt{2 n+\frac{7}{2}}}\left(1+\delta_{1} e^{t \delta \sqrt{2 n+\frac{3}{2}}}\right)}{\left(1+\delta_{2}\left(2 n+\frac{7}{2}\right)\right) e^{\delta t \sqrt{2 n+\frac{3}{2}}}\left(1+\delta_{1} e^{t \delta \sqrt{2 n+\frac{7}{2}}}\right)}\right. \\
& +\frac{\left(1+\delta_{2}\left(2 n+\frac{7}{2}\right)\right) e^{\delta t \sqrt{2 n+\frac{3}{2}}}\left(1+\delta_{1} e^{t \delta \sqrt{2 n+\frac{7}{2}}}\right)}{\left(1+\delta_{2}\left(2 n+\frac{3}{2}\right)\right) e^{\delta t \sqrt{2 n+\frac{7}{2}}}\left(1+\delta_{1} e^{\left.t \delta \sqrt{2 n+\frac{3}{2}}\right)}\right]} \\
& \geq \sum_{n=2}^{\infty} 8 n(2 n+3)\left|h_{n, \delta_{2}}(t)\right|^{2}-\sum_{n=2}^{\infty} 8 n \sqrt{(n+1)\left(n+\frac{3}{2}\right)}\left|h_{n, \delta_{2}}(t)\right|\left|h_{n+1, \delta_{2}}(t)\right| \mathbf{R}
\end{aligned}
$$

where

$$
\mathbf{R}=\frac{e^{\delta t \sqrt{2 n+\frac{7}{2}}}\left(1+\delta_{1} e^{t \delta \sqrt{2 n+\frac{3}{2}}}\right)}{e^{\delta t \sqrt{2 n+\frac{3}{2}}}\left(1+\delta_{1} e^{t \delta \sqrt{2 n+\frac{7}{2}}}\right)}+\left(\frac{2 n+\frac{7}{2}}{2 n+\frac{3}{2}}\right) \frac{e^{\delta t \sqrt{2 n+\frac{3}{2}}}\left(1+\delta_{1} e^{t \delta \sqrt{2 n+\frac{7}{2}}}\right)}{e^{\delta t \sqrt{2 n+\frac{7}{2}}}\left(1+\delta_{1} e^{t \delta \sqrt{2 n+\frac{3}{2}}}\right)}
$$

Direct calculation shows that, for any $0<t \leq T$

$$
\begin{aligned}
\mathbf{R} & \leq \frac{2}{2 n+3 / 2}+e^{\frac{2 \delta t}{\sqrt{2 n+3 / 2}+\sqrt{2 n+7 / 2}}}+e^{\frac{-2 \delta t}{\sqrt{2 n+3 / 2}+\sqrt{2 n+7 / 2}}} \\
& \leq 2+\frac{4}{4 n+3}+\frac{\delta^{2} t^{2}}{2 n+3 / 2}\left(e^{\frac{2 \delta \tau}{\sqrt{2 n+3 / 2}+\sqrt{2 n+7 / 2}}}+e^{\frac{-2 \delta \tau}{\sqrt{2 n+3 / 2}+\sqrt{2 n+7 / 2}}}\right) \\
& \leq 2+\frac{1}{n+3 / 4}\left(1+\delta^{2} T^{2} e^{\frac{\delta T}{2}}\right) .
\end{aligned}
$$

Let $\delta=\frac{1}{\sqrt{2} T}$, since $n+3 / 4 \geq \sqrt{n(n+3 / 2)}$, we have

$$
\mathbf{R} \leq 2+\frac{2}{\sqrt{n(n+3 / 2)}}
$$


Then it follows from the Cauchy-Schwartz inequality that

$$
\begin{aligned}
& \left(\left(1+\delta_{2} \mathcal{H}\right)^{-1}\left(M_{\delta_{1}}(\delta t)\right) \mathcal{L}\left(\left(M_{\delta_{1}}(\delta t)\right)^{-1} h\right), h_{\delta_{2}}\right) \\
& \geq \sum_{n=2}^{\infty} 8 n(2 n+3)\left|h_{n, \delta_{2}}(t)\right|^{2}-2 \sum_{n=2}^{\infty} 8 n \sqrt{(n+1)\left(n+\frac{3}{2}\right)}\left|h_{n, \delta_{2}}(t)\right|\left|h_{n+1, \delta_{2}}(t)\right| \\
& -2 \sum_{n=2}^{\infty} 8 \sqrt{n(n+1)}\left|h_{n, \delta_{2}}(t)\right|\left|h_{n+1, \delta_{2}}(t)\right| \\
& \geq \sum_{n=2}^{\infty} 4 n(2 n+3)\left|h_{n, \delta_{2}}(t)\right|^{2}-\sum_{n=2}^{\infty} 4 n(2 n+2)\left|h_{n+1, \delta_{2}}(t)\right|^{2} \\
& -\sum_{n=2}^{\infty} 8 n\left|h_{n, \delta_{2}}(t)\right|^{2}-\sum_{n=2}^{\infty} 8(n+1)\left|h_{n+1, \delta_{2}}(t)\right|^{2} \\
& =\sum_{n=2}^{\infty} 4 n\left|h_{n, \delta_{2}}(t)\right|^{2} \geq\left\|\mathcal{H}^{\frac{1}{2}} h_{\delta_{2}}\right\|_{L^{2}}^{2} \text {. }
\end{aligned}
$$

Remind that $h \in \mathcal{N}^{\perp}$, we can deduce from Proposition 2.1 that

$$
\begin{aligned}
& \left(\left(1+\delta_{2} \mathcal{H}\right)^{-1}\left(M_{\delta_{1}}(\delta t)\right) \Gamma\left(\left(M_{\delta_{1}}(\delta t)\right)^{-1} h,\left(M_{\delta_{1}}(\delta t)\right)^{-1} h\right), h_{\delta_{2}}\right) \\
= & \frac{\left(1+\delta_{2}\left(2 n+\frac{3}{2}\right)\right)\left(1+\frac{11}{2} \delta_{2}\right)}{1+\delta_{2}\left(2 n+\frac{7}{2}\right)} \sum_{n=2}^{\infty} \frac{8}{3} \sqrt{30(n+1)\left(n+\frac{3}{2}\right)} \\
& \times \frac{\left(1+\delta_{1} e^{\sqrt{\frac{11}{2}} \delta t}\right)\left(1+\delta_{1} e^{\delta t} \sqrt{2 n+\frac{3}{2}}\right) e^{\delta t \sqrt{2 n+\frac{7}{2}}}}{e^{\delta t \sqrt{2 n+\frac{3}{2}}}} h^{\sqrt{\frac{11}{2} \delta t}}\left(1+\delta_{1} e^{\delta t \sqrt{2 n+\frac{3}{2}}}\right)
\end{aligned}
$$

Since

$$
e^{\delta t \sqrt{2 n+\frac{7}{2}}} \leq e^{\delta t \sqrt{2 n+\frac{3}{2}}} e^{\delta t \sqrt{\frac{11}{2}}}
$$

recall that $\delta_{1}, \delta_{2} \leq 1$, we have

$$
\begin{aligned}
& \left|\left(\left(1+\delta_{2} \mathcal{H}\right)^{-1}\left(M_{\delta_{1}}(\delta t)\right) \Gamma\left(\left(M_{\delta_{1}}(\delta t)\right)^{-1} h,\left(M_{\delta_{1}}(\delta t)\right)^{-1} h\right), h_{\delta_{2}}\right)\right| \\
\leq & \left(1+3 \delta_{1}+3 \delta_{2}\right) \sum_{n=2}^{\infty} \frac{8}{3} \sqrt{30(n+1)\left(n+\frac{3}{2}\right)}\left|h_{2, \delta_{2}}(t)\right|\left|h_{n, \delta_{2}}(t)\right|\left|h_{n+1, \delta_{2}}(t)\right| \\
\leq & C\left\|\mathbb{P}_{2} h_{\delta_{2}}(t)\right\|_{L^{2}}\left\|\mathcal{H}^{\frac{1}{2}} h_{\delta_{2}}\right\|_{L^{2}}^{2} .
\end{aligned}
$$

Substituting the estimates (3.5) and (3.6) into (3.4), one can verify that

$$
\frac{\mathrm{d}}{\mathrm{d} t}\left\|h_{\delta_{2}}\right\|_{L^{2}}^{2}+2\left\|\mathcal{H}^{\frac{1}{2}} h_{\delta_{2}}\right\|_{L^{2}}^{2} \leq C\left\|\mathbb{P}_{2} h_{\delta_{2}}(t)\right\|_{L^{2}}\left\|\mathcal{H}^{\frac{1}{2}} h_{\delta_{2}}\right\|_{L^{2}}^{2}+\delta\left\|\mathcal{H}^{\frac{1}{2}} h_{\delta_{2}}\right\|_{L^{2}}^{2}
$$

We consider the fact that,

$$
\|g\|_{L^{2}} \leq\left\|g_{0}\right\|_{L^{2}} \leq \epsilon_{0}
$$


then for $0<t \leq T$ and $\delta \leq \frac{1}{\sqrt{2} T}$,

$$
\left\|\mathbb{P}_{2} h_{\delta_{2}}(t)\right\|_{L^{2}}^{2} \leq e^{2 \delta T \sqrt{\frac{11}{2}}}\left|g_{2}\right|^{2} \leq e^{4} \epsilon_{0}^{2} .
$$

Therefore, choose $\epsilon, \delta$ small, we have

$$
\frac{\mathrm{d}}{\mathrm{d} t}\left\|h_{\delta_{2}}\right\|_{L^{2}}^{2}+\left\|\mathcal{H}^{\frac{1}{2}} h_{\delta_{2}}\right\|_{L^{2}}^{2} \leq 0 .
$$

This means that, for any $0<t \leq T$,

$$
\left\|h_{\delta_{2}}(t)\right\|_{L^{2}}^{2}+\int_{0}^{t}\left\|\mathcal{H}^{\frac{1}{2}} h_{\delta_{2}}(\tau)\right\|_{L^{2}}^{2} \mathrm{~d} \tau \leq\left\|g_{0}\right\|_{L^{2}}^{2} .
$$

Let $\delta_{2} \rightarrow 0$, we have

$$
\left\|e^{\delta t \sqrt{\mathcal{H}}} g(t)\right\|_{L^{2}}^{2}+\int_{0}^{t}\left\|\mathcal{H}^{\frac{1}{2}} e^{\delta t \sqrt{\mathcal{H}}} g(\tau)\right\|_{L^{2}}^{2} \mathrm{~d} \tau \leq\left\|g_{0}\right\|_{L^{2}}^{2} .
$$

This ends the proof of Theorem 1.1.

\section{The proof of Proposition 2.1}

In this section, we will prove the proposition 2.1. This Proposition shows the spectral decomposition for the linear and nonlinear radially symmetric spatially homogeneous Landau operators under the hard potential $\gamma=2$ in details. At the beginning, we introduce the kronecker function

$$
\delta_{n, m}= \begin{cases}1, & m=n, \\ 0, & m \neq n .\end{cases}
$$

Proposition 4.1. Let $\Gamma$ be the nonlinear Landau operator, the following algebraic identities hold true, for $n, m \in \mathbb{N}$

$$
\begin{aligned}
\Gamma\left(\varphi_{n}, \varphi_{m}\right)=\frac{1}{\sqrt{\mu(v)}} & {\left[10\left(|v|^{2}+3\right)\left(\Psi_{m}\left(\frac{|v|^{2}}{2}\right)+\Psi_{m}^{\prime}\left(\frac{|v|^{2}}{2}\right)\right)\right.} \\
+ & \left.2|v|^{2}\left(|v|^{2}+5\right)\left(\Psi_{m}\left(\frac{|v|^{2}}{2}\right)+\Psi_{m}^{\prime}\left(\frac{|v|^{2}}{2}\right)\right)^{\prime}\right] \delta_{0, n} \\
& -\frac{2 \sqrt{6}}{3 \sqrt{\mu(v)}}\left[15 \Psi_{m}\left(\frac{|v|^{2}}{2}\right)+10\left(|v|^{2}+3\right) \Psi_{m}^{\prime}\left(\frac{|v|^{2}}{2}\right)\right. \\
+ & \left.|v|^{2}\left(|v|^{2}+10\right) \Psi_{m}^{\prime \prime}\left(\frac{|v|^{2}}{2}\right)\right] \delta_{1, n}
\end{aligned}
$$




$$
+\frac{2 \sqrt{120}}{3 \sqrt{\mu(v)}}\left[3 \Psi_{m}^{\prime}\left(\frac{|v|^{2}}{2}\right)+|v|^{2} \Psi_{m}^{\prime \prime}\left(\frac{|v|^{2}}{2}\right)\right] \delta_{2, n} .
$$

Proof. In all the proof of this proposition, we will set $\Psi_{k}: \mathbb{R} \rightarrow \mathbb{R}$

$$
\Psi_{n}(\rho)=c_{n} L_{n}^{\left(\frac{1}{2}\right)}(\rho) e^{-\rho}
$$

with

$$
c_{n}=(2 \pi)^{-\frac{3}{4}} \sqrt{\frac{n !}{4 \sqrt{2} \pi \Gamma\left(n+\frac{3}{2}\right)}} .
$$

Therefore, recalled from the definition of $\varphi_{n}(v)$ that, for $v \in \mathbb{R}^{3}$,

$$
\sqrt{\mu}(v) \varphi_{n}(v)=c_{n} L_{n}^{\left(\frac{1}{2}\right)}\left(\frac{|v|^{2}}{2}\right) e^{-\frac{|v|^{2}}{2}}=\Psi_{n}\left(\frac{|v|^{2}}{2}\right) .
$$

It follows that, for $m, n \in \mathbb{N}$

$$
\nabla_{v_{*}}\left(\sqrt{\mu} \varphi_{n}\right)\left(v_{*}\right)=\Psi_{n}^{\prime}\left(\frac{\left|v_{*}\right|^{2}}{2}\right) v_{*}, \quad \nabla_{v}\left(\sqrt{\mu} \varphi_{m}\right)(v)=\Psi_{m}^{\prime}\left(\frac{|v|^{2}}{2}\right) v,
$$

where we used the notation $\Psi_{k}(\rho)$ in (4.1) and $\Psi_{k}^{\prime}(\rho)=\frac{d \Psi_{k}(\rho)}{d \rho}$ for $k \in \mathbb{N}$. Then

$$
\begin{aligned}
\Gamma\left(\varphi_{n}, \varphi_{m}\right)=\frac{1}{\sqrt{\mu(v)}} \sum_{1 \leq i, j \leq 3} \partial_{v_{i}} \int_{\mathbb{R}^{3}} a_{i, j}\left(v-v_{*}\right) \\
\quad \times\left[\Psi_{n}\left(\frac{\left|v_{*}\right|^{2}}{2}\right) \Psi_{m}^{\prime}\left(\frac{|v|^{2}}{2}\right) v_{j}-\Psi_{n}^{\prime}\left(\frac{\left|v_{*}\right|^{2}}{2}\right) \Psi_{m}\left(\frac{|v|^{2}}{2}\right) v_{j}^{*}\right] \mathrm{d} v_{* \prime}
\end{aligned}
$$

where we have written $v=\left(v_{1}, v_{2}, v_{3}\right), v_{*}=\left(v_{1}^{*}, v_{2}^{*}, v_{3}^{*}\right) \in \mathbb{R}^{3}$ and

$$
\begin{aligned}
& a_{i, i}\left(v-v_{*}\right)=\sum_{\substack{1 \leq k \leq 3 \\
k \neq i}}\left(v_{k}-v_{k}^{*}\right)^{2}\left|v-v_{*}\right|^{2} ; \\
& a_{i, j}\left(v-v_{*}\right)=-\left(v_{i}-v_{i}^{*}\right)\left(v_{j}-v_{j}^{*}\right)\left|v-v_{*}\right|^{2}, \quad \text { when } i \neq j .
\end{aligned}
$$

Direct computation shows that

$$
\begin{aligned}
& \quad \boldsymbol{\Gamma}\left(\varphi_{n}, \varphi_{m}\right) \\
& =\frac{1}{\sqrt{\mu(v)}} \sum_{\substack{1 \leq i, j \leq 3 \\
i \neq j}} \partial_{v_{i}}\left\{\int _ { \mathbb { R } ^ { 3 } } \left[v_{i}\left(v_{j}^{*}\right)^{2}|v|^{2}+2 v_{i} v_{j}^{2}\left(v_{j}^{*}\right)^{2}\right.\right. \\
& \left.\left.\quad+v_{i}\left(v_{j}^{*}\right)^{2}\left|v_{*}\right|^{2}-2 v_{j}^{2} v_{i}\left(v_{i}^{*}\right)^{2}\right] \Psi_{n}\left(\frac{\left|v_{*}\right|^{2}}{2}\right) \mathrm{d} v_{*} \Psi_{m}^{\prime}\left(\frac{|v|^{2}}{2}\right)\right\}
\end{aligned}
$$




$$
\begin{aligned}
& +\frac{1}{\sqrt{\mu(v)}} \sum_{\substack{\leq \leq, j, \leq 3 \\
i \neq j}} \partial_{v_{i}}\left\{\int _ { \mathbb { R } ^ { 3 } } \left[2 v_{i} v_{j}^{2}\left(v_{i}^{*}\right)^{2}-2 v_{i} v_{j}^{2}\left(v_{j}^{*}\right)^{2}\right.\right. \\
& \left.\left.-v_{i}\left(v_{j}^{*}\right)^{2}|v|^{2}-v_{i}\left(v_{j}^{*}\right)^{2}\left|v_{*}\right|^{2}\right] \Psi_{n}^{\prime}\left(\frac{\left|v_{*}\right|^{2}}{2}\right) \mathrm{d} v_{*} \Psi_{m}\left(\frac{|v|^{2}}{2}\right)\right\} .
\end{aligned}
$$

By using the elementary equalities

$$
\begin{aligned}
& \sqrt{\mu}\left(v_{*}\right)=\varphi_{0}, \\
& \left|v_{*}\right|^{2} \sqrt{\mu}\left(v_{*}\right)=3 \varphi_{0}-\sqrt{6} \varphi_{1}, \\
& \left|v_{*}\right|^{4} \sqrt{\mu}\left(v_{*}\right)=15 \varphi_{0}-10 \sqrt{6} \varphi_{1}+\sqrt{120} \varphi_{2},
\end{aligned}
$$

we can deduce from $\Psi_{n}\left(\frac{\left|v_{*}\right|^{2}}{2}\right)=\sqrt{\mu}\left(v_{*}\right) \varphi_{n}\left(v_{*}\right)$ that

$$
\begin{aligned}
& \int_{\mathbb{R}^{3}} \Psi_{n}\left(\frac{\left|v_{*}\right|^{2}}{2}\right) \mathrm{d} v_{*}=\delta_{0, n}, \\
& \int_{\mathbb{R}^{3}}\left|v_{*}\right|^{2} \Psi_{n}\left(\frac{\left|v_{*}\right|^{2}}{2}\right) \mathrm{d} v_{*}=3 \delta_{0, n}-\sqrt{6} \delta_{1, n}, \\
& \int_{\mathbb{R}^{3}}\left|v_{*}\right|^{4} \Psi_{n}\left(\frac{\left|v_{*}\right|^{2}}{2}\right) \mathrm{d} v_{*}=15 \delta_{0, n}-10 \sqrt{6} \delta_{1, n}+\sqrt{120} \delta_{2, n} .
\end{aligned}
$$

Similar to the above symmetric property, and integration by parts, we can also prove that

$$
\int_{\mathbb{R}^{3}}\left|v_{*}\right|^{2} \Psi_{n}^{\prime}\left(\frac{\left|v_{*}\right|^{2}}{2}\right) \mathrm{d} v_{*}=-3 \int_{\mathbb{R}^{3}} \sqrt{\mu}\left(v_{*}\right) \varphi_{n}\left(v_{*}\right) \mathrm{d} v_{*}=-3 \delta_{0, n}
$$

and

$$
\int_{\mathbb{R}^{3}}\left|v_{*}\right|^{4} \Psi_{n}^{\prime}\left(\frac{\left|v_{*}\right|^{2}}{2}\right) \mathrm{d} v_{*}=-5\left(3 \delta_{0, n}-\sqrt{6} \delta_{1, n}\right) .
$$

By using the symmetric of the coordinate axis, we end the proof of Prop. 4.1.

Now we prepare to prove the Proposition 2.1.

The proof of Proposition 2.1. Denote that

$$
\begin{aligned}
& \mathbf{A}(v)=10\left(|v|^{2}+3\right)\left(\Psi_{m}\left(\frac{|v|^{2}}{2}\right)+\Psi_{m}^{\prime}\left(\frac{|v|^{2}}{2}\right)\right) \\
& +2|v|^{2}\left(|v|^{2}+5\right)\left(\Psi_{m}\left(\frac{|v|^{2}}{2}\right)+\Psi_{m}^{\prime}\left(\frac{|v|^{2}}{2}\right)\right)^{\prime} \\
& \mathbf{B}(v)=15 \Psi_{m}\left(\frac{|v|^{2}}{2}\right)+10\left(|v|^{2}+3\right) \Psi_{m}^{\prime}\left(\frac{|v|^{2}}{2}\right)+|v|^{2}\left(|v|^{2}+10\right) \Psi_{m}^{\prime \prime}\left(\frac{|v|^{2}}{2}\right),
\end{aligned}
$$




$$
\mathbf{C}(v)=3 \Psi_{m}^{\prime}\left(\frac{|v|^{2}}{2}\right)+|v|^{2} \Psi_{m}^{\prime \prime}\left(\frac{|v|^{2}}{2}\right)
$$

Recall from the definition of $\Psi_{m}\left(\frac{|v|^{2}}{2}\right)$ in (4.1) and take intermediate variable $\rho=\frac{|v|^{2}}{2}$, we have

$$
\begin{aligned}
& \Psi_{m}\left(\frac{|v|^{2}}{2}\right)=c_{m} e^{-\rho} L_{m}^{\left(\frac{1}{2}\right)}(\rho) \\
& \Psi_{m}^{\prime}\left(\frac{|v|^{2}}{2}\right)=c_{m} e^{-\rho} \frac{\mathrm{d}}{\mathrm{d} \rho} L_{m}^{\left(\frac{1}{2}\right)}(\rho)-c_{m} e^{-\rho} L_{m}^{\left(\frac{1}{2}\right)}(\rho), \\
& \Psi_{m}^{\prime \prime}\left(\frac{|v|^{2}}{2}\right)=c_{m} e^{-\rho} \frac{\mathrm{d}^{2}}{\mathrm{~d}^{2} \rho} L_{m}^{\left(\frac{1}{2}\right)}(\rho)-2 c_{m} e^{-\rho} \frac{\mathrm{d}}{\mathrm{d} \rho} L_{m}^{\left(\frac{1}{2}\right)}(\rho)+c_{m} e^{-\rho} L_{m}^{\left(\frac{1}{2}\right)}(\rho) .
\end{aligned}
$$

Using the formulas $\left(14_{1}\right),(7),(12)$ of Chapter IV in [14] that, we have

$$
\begin{aligned}
& \mathbf{A}(v)=8 m \sqrt{(m+1)\left(m+\frac{3}{2}\right)} \Psi_{m+1}-8 m(2 m+3) \Psi_{m}+8(m-1) \sqrt{m\left(m+\frac{1}{2}\right)} \Psi_{m-1}, \\
& \mathbf{B}(v)=4 \sqrt{(m+1)(m+2)\left(m+\frac{3}{2}\right)\left(m+\frac{5}{2}\right)} \Psi_{m+2} \\
& \quad-4(2 m+5) \sqrt{(m+1)\left(m+\frac{3}{2}\right)} \Psi_{m+1}+4 m(m-1) \Psi_{m}, \\
& \mathbf{C}(v)=-2 \sqrt{(m+1)\left(m+\frac{3}{2}\right)} \Psi_{m+1} .
\end{aligned}
$$

Substituting back to Proposition 4.1, we end the proof of Proposition 2.1.

\section{Acknowledgement}

The authors would like to express their sincere thanks to Prof. Chao-Jiang $\mathrm{Xu}$ for his stimulating suggestions. This research is supported by the Fundamental Research Fund$s$ for the Central Universities of China, South-Central University for Nationalities (No. CZT20007) and the Natural Science Foundation of China (No. 11701578).

\section{References}

[1] Villani C., On a new class of weak solutions to the spatially homogeneous Boltzmann and Landau equations. Arch. Rational Mech. Anal., 143 (1998), 273-307.

[2] Villani C., On the spatially homogeneous Landau equation for Maxwellian molecules. Mathematical Models and Methods in Applied Sciences, 8 (1998), 957-983.

[3] Gramchev T., Pilipović S. and Rodino L., Classes of degenerate elliptic operators in GelfandShilov spaces. New Developments in Pseudo-Differential Operators. Birkhäuser Basel, (2009), 15-31. 
[4] Toft J., Khrennikov A., Nilsson B. and Nordebo S., Decompositions of Gelfand-Shilov kernels into kernels of similar class. J. Math. Anal. Appl., 396 (1) (2012), 315-322.

[5] Desvillettes L., Villani C., On the spatially homogeneous Landau equation for hard potentials. I. Existence, uniqueness and smoothness. Comm. Partial Differential Equations, 25 (8) (2000), 179-259.

[6] Guo Y., The Landau equation in a periodic box. Comm. Math. Phys., 231 (2002), 391-434.

[7] Chen H., Li W.-X. and Xu C.-J., Analytic smoothness effect of solutions for spatially homogeneous Landau eqaution. J. Differential Equations, 248 (2010) , 77-94.

[8] Chen H., Li W.-X. and Xu C.-J., Propagation of Gevrey regularity for solutions of Landau equations. Kinet. Related Models, 1 (2008), 355- 368.

[9] Lerner N., Morimoto Y., Pravda-Starov K. and Xu C.-J., Spectral and phase space analysis of the linearized non-cutoff Kac collision operator. J. Math. Pures Appl., 100 (6) (2013), 832-867.

[10] Lerner N., Morimoto Y., Pravda-Starov K. and Xu C.-J., Phase space analysis and functional calculus for the linearized Landau and Boltzmann operators. Kinet. Relat. Models, 6 (3) (2013), 625-648.

[11] Lerner N., Morimoto Y., Pravda-Starov K. and Xu C.-J., Gelfand-Shilov smoothing properties of the radially symmetric spatially homogeneous Boltzmann equation without angular cutoff. J. Differential Equations, 256 (2) (2014), 797-831.

[12] Morimoto Y., Pravda-Starov K. and Xu C.-J., A remark on the ultra-analytic smoothing properties of the spatially homogeneous Landau equation. Kinet. Related Models, 6 (4) (2013), 715727.

[13] Li H.-G., Xu C.-J., Cauchy problem for the spatially homogeneous landau equation with shubin class initial datum and Gelfand-Shilov smoothing effect. SIAM J. Math. Anal., 51 (2019), 532-564.

[14] Sansone G., Orthogonal Functions, Pure and Applied Mathematics, Vol. IX, 1959. 